\title{
RIGHT-SIDED COLITIS
}

\author{
By E. C. B. Butler, F.R.C.S.
}

The London Hospital

\section{Definition}

Infections of the colon are notoriously difficult to classify since their etiology is generally obscure. In the past it was customary to group all chronic inflammations of the large bowel under one heading, ulcerative colitis.

During the past ten years attempts have been made by many workers to subdivide up infections of the colon based either on the anatomical situation of the infection or on the pathology.

We believe that this division is very important since the surgical treatment of these infections is essentially different from that of true ulcerative colitis.

In 1947 Crohn, Garlock and Yarnis first described right-sided colitis and they were of the opinion that this group was responsible for about 8 per cent. of colonic infections.

Brooke (1954), on the other hand, while agreeing that this group is dissimilar from ulcerative colitis maintains that the infection invariably starts in the ileum and then spreads distally to affect a variable amount of the large bowel.

In my view both types of infection occur but I agree with Brooke that it is often difficult even at operation to distinguish one from the other.

\section{Types of Infection}

It is possible to differentiate the following types of right-sided colitis :

I. The infection starts in the right colon and spreads distally sometimes invading the whole of the large bowel. The small intestine is not affected. Histologically this group resembles regional rather than idiopathic ulcerative colitis.

2. Similar to the above but with involvement of the distal ileum; this group may be regarded as regional ileitis which has spread into the right colon since the pathological appearances of the small and large gut are similar to these described by Crohn.

3. Segmental infection of the right colon which shows little tendency to spread and has normal bowel on either side of the affected segment.

4. True ileo colitis as described by Brooke; the infection of the small gut is often irregular in distribution and may affect a large amount of the small intestine. The typical thickening of the ileum as seen in Crohn's disease is absent and the amount of large bowel involvement is very variable.

5. Tubercular infection of the right colon and ileum undoubtedly occurs but will not be dealt with in this paper.

\section{Group I}

This group is characterized by a long-standing history of intermittent diarrhoea, wasting and pus in the stools. There is generally an irregular pyrexia but blood in the motions is unusual. Toxaemia may occur and polyarthritis is not uncommon.

Diagnosis. Sigmoidoscopy reveals a normal rectum and lower pelvic colon and a barium enema shows infection of a variable amount of the right colon. A follow meal reveals no ileal disease. In time the infection may spread slowly into the distal colon.

Treatment. As soon as the diagnosis is established surgery should be advised in order to prevent distal spread of the infection. Medical treatment has little influence in this group.

The object of the treatment is either to isolate the affected bowel and later remove it or to carry out an immediate subtotal colectomy with implantation of the normal ileum into the pelvic colon. In my experience the two-stage operation is usually the safest. At the first operation the lower ileum is divided; the distal end closed and the proximal end anastamosed to normal pelvic colon. The upper pelvic colon is also divided, the proximal end brought out as a mucous fistula and the distal end closed.

It is essential to divide the pelvic colon as otherwise the infection might creep down into the pelvic colon and later invade the ileum via the anastamosis.

\section{Case I}

A woman aged 35 . She had a history of II 
months' diarrhoea, intermittent pyrexia and loss of weight. On examination a wasted ill woman with an irregular pyrexia. A barium enema showed signs of infection of the proximal colon as far as the lower end of the descending colon. Sigmoidoscopy was normal. Operation, August 20, 1947 .

Laparotomy, chronic inflammation of the whole colon from the caecum to the beginning of the pelvic colon. The ileum was normal. The affected bowel was isolated and drained by a mucous fistula and the ileum was joined to the pelvic colon.

The patient's clinical state improved dramatically after the operation and she has remained well to date. She has never had her diseased colon removed as she is unwilling to undergo further surgery.

The interesting points in her case were: No infection of the ileum; the marked demarcation between healthy and diseased large bowel; the rapid improvement which followed isolation and drainage of the diseased segment.

\section{Group 2}

This group is more frequent than the last. The symptoms are identical. The only diagnostic difference is that a follow through meal may show infection of the distal ileum:

The principles of treatment are identical but care must be taken to divide the ileum well above the area of inflammation in order to avoid, if possible, a recurrence in the proximal small bowel.

It is impossible to say in most of these cases where the infection started. It may have originated in the small gut and spread distally or alternatively have invaded the ileum via an incompetent ileo caecal valve.

\section{Case 2}

Woman aged 22. Nearly two years' abdominal pain and diarrhoea. On admission she was a wasted woman with a palpable caecum. Sigmoidoscopy was normal and a barium enema showed infection of the right colon as far as the splenic flexure. A follow through meal demonstrated disease of the terminal ileum.

A course of ACTH was commenced on May 2, 1952, with immediate clinical improvement but a relapse occurred as soon as the drug was stopped.

Laparotomy, October 8, 1952. The terminal ileum to halfway down the descending colon was involved by chronic infection. An exclusion operation was carried out and later the affected bowel was excised.

Pathological report. Regional colitis chiefly affecting the right colon and terminal ileum:
This patient has remained well and has returned to work.

\section{Case 3}

Woman aged 32. Two months' history of pyrexia, abdominal pain and diarrhoea., A barium enema showed right-sided colitis extending to the middle of the descending colon. Sigmoidoscopy was normal.

Laparotomy, April 25, 1951. The terminal ileum and all the large bowel down to the pelvic colon was involved by infection. The lymphatic nodes were large and juicy and there was no clear line of demarcation between diseased and healthy bowel. The affected bowel was excluded.

Readmitted for partial colectomy, December 30, I95I.

Pathological report. Ileitis and colitis probably Crohn's disease. This patient also has remained well since her last operation. Both these patients seem to have had an ileitis and colitis as described by Crohn and they have responded well to a two-stage surgical excision.

\section{Segmental colitis}

These infections differ from the last two groups as the lesions appear localized and there is normal bowel on either side. Separate segments of the large gut may be affected at the same or different times and the diagnosis from carcinoma, tuberculosis or diverticulosis is usually difficult.

The symptomatology includes diarrhoea, wasting, anaemia and abdominal mass or intestinal obstruction.

This group is rare in the right colon but $I$ have met with two cases. Treatment is surgical and a laparotomy is often required to establish a definite diagnosis.

\section{Case 4}

Woman aged 43. In 1944 excision of the pelvic colon for supposed carcinoma elsewhere. Well since. Lately she had complained of right-sided abdominal pain. On examination, tender lump in the right iliac fossa and a barium enema showed a filling defect in the caecum.

Laparotomy, September 10; 1952, showed a hard lump in the caecum which was thought to be a carcinoma. Right hemi-colectomy performed.

Pathological report. Chronic granulomatous infection of all coats of the caecum allied to Crohn's disease. Following this report we wrote to the hospital where the first operation had been carried out. They looked up their report and found it was not a carcinoma but an area of colitis in the pelvic colon.

Here we have an example of a patient developing two separate areas of segmental colitis in different 


\section{LLOYD-LUKE}

\section{Books that enshrine profound thought}

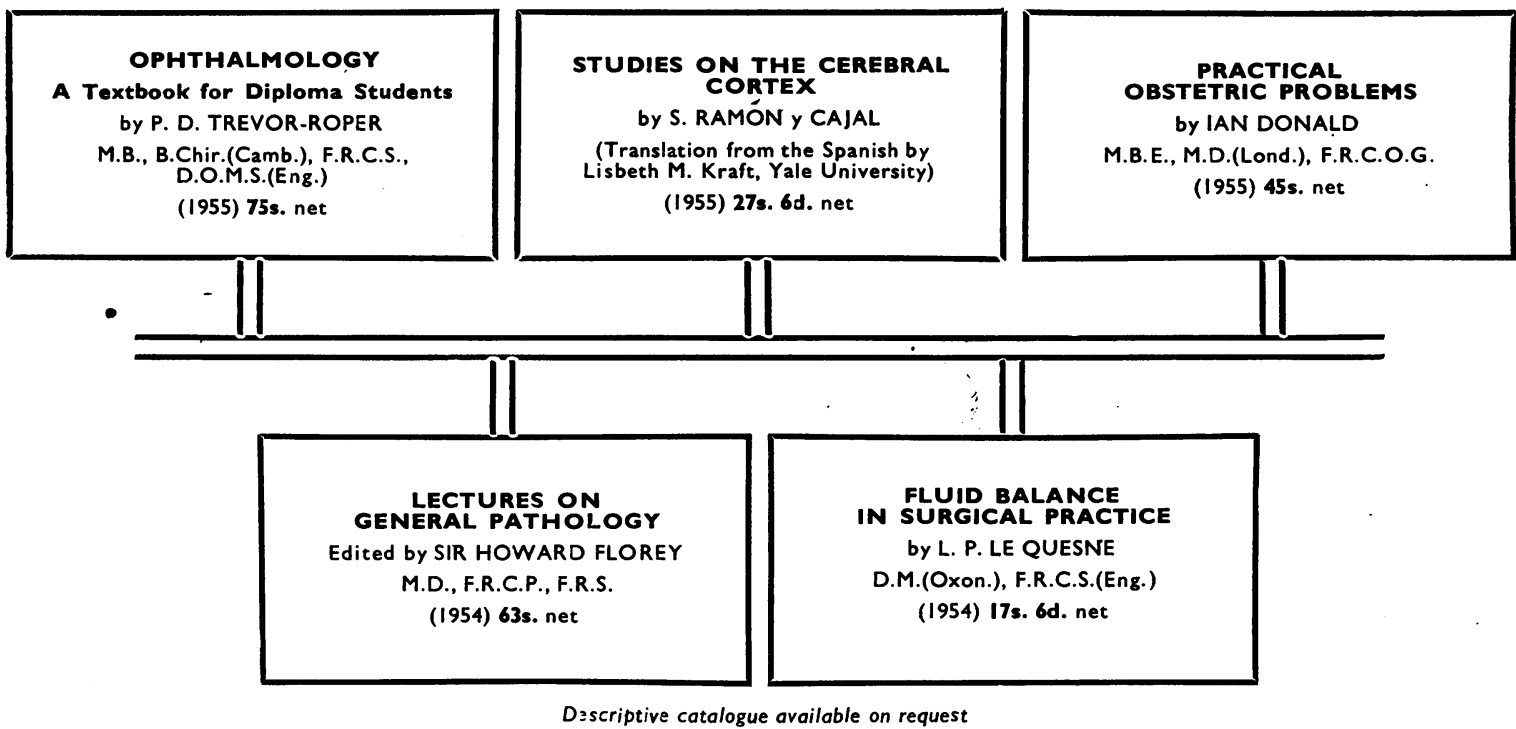

\section{LLOYD-LUKE (Medical вoOKs) LTD., 49 Newman Street, W.I}

parts of the colon at widely separated intervals. In both cases a diagnosis of carcinoma was made.

\section{Group 4}

True Ileo Colitis

This condition is well described by Brooke but I have never met with one to my knowledge. The disease is said to start as a steatorrhea and then to proceed to the symptoms of an ileo colitis. A follow through meal shows infection of the ileum but this does not follow a regular pattern and the lesions may involve a considerable amount of the small intestine.

Surgical treatment is difficult since it is rarely possible to identify with certainty the upper limit of the infection, even at operation. These patients are very liable to be diagnosed as ulcerative colitis and the end results are likely to be disastrous should an ileostomy be carried out.

\section{Conclusions}

I. There are at least four different kinds of chronic infection of the right colon: (a) Right-sided colitis with a normal ileum.

(b) Right-sided colitis with an infected ileum.

(c) Segmental colitis.

(d) Diffuse ileo colitis.

2. Ileostomy should never be carried out on any of these types of infection.

3. Types (a) and (b) should be treated by a two-stage excision with implantation of the normal ileum into the pelvic colon.

4. Type (c) should be treated by right hemicolectomy.

5. Type (d), if recognized, should be treated by general medical measures.

6 . The diagnostic criterion in all these cases is that the pelvic colon and rectum are free from disease when examined by a sigmoidoscope.

My thanks are due to the Editors of the Proceedings of the Royal Society of Medicine for their permission to reproduce a good deal of this article.

\section{BIBLIOGRAPHY}

CROHN, B. B., GARLOCK, J. H. and YARNIS, H. (1947), F. Amer. med. Ass., 134, 334.

BUTLER, E. C. B. (1953), Proc. R.S. Med. 46, 2, 65. BROOKE (r954), 'Ulcerative Colitis and its Treatment.' 